\title{
Use of homeopathic nux vomica in reducing PAWS (post acute withdrawal syndrome) in early recovering addicted women
}

\section{Introduction}

Setting: Women's Circle Recovery House (Cohort 1) 26-28 Thane St, Dorchester MA, 02121 Griffin House Recovery House (Cohort 2) 26-28 Thane St, Dorchester MA, 02121,, Kitty Dukakis Treatment Center Hopefound, Inc (Cohort 3) 170 Morton Street, Jamaica Plain, MA 02130

A. Context: Homeopathic remedy Nux vomica is commonly used to alleviate symptoms of excessive alcohol and other drug use.

B. Objective: To assess whether Nux vomica administered post detox effects occurrence of post acute withdrawal symptoms (PAWS) in recovering addicted persons.

C. Design: Cohort comparisons-those chosing to use homeopathy and those choosing not to use comparing completion of program outcomes.

D. Setting: Residential treatment for women with substance abuse addiction, Boston MA

E. Participants: Three separate cohorts (2006-2011) those using homeopathy and those choosing not to use homeopathy. Women between ages of 18 and 60, varied ethnicity and all multiple drug users with addiction.

F. Intervention: Administered 200c Nux vomica (single dose) post detox for continued cravings for drugs, and symptoms of irritability, sleeplessness and other symptoms of PAWS.

G. Main Outcome Measures: Successful completion of substance abuse treatment program (6months for 2 cohorts, 28 days for $3 \mathrm{rd}$ cohort.)

H. Results: Consistently in all three groups, those choosing to use homeopathy completed substance abuse treatment programs at a higher rate than those who did not.

I. Conclusions: The role of homeopathic for recovery from addiction merits further investigation.

\section{Homeopathy}

Classical homeopathy is a 200-year-old system of healing developed by the German physician Samuel Hahnemann. It is holistic and works with the person's natural ability to heal.

The action of a homeopathic remedy is understood by testing it on healthy individuals, observing symptoms produced and thus compiling a "remedy picture." This is a process known as a homeopathic proving. When someone presents with a health issue, their symptom picture (mental- emotional-and physical) is then correlated to a remedy picture, and that remedy is given. The remedies
Volume 6 Issue 4 - 2017

\author{
Loretta Butehorn,' Peris Gumz, ${ }^{2}$ Lead \\ Randolph ${ }^{3}$ \\ 'Department of Public Health, Boston College, USA \\ ${ }^{2}$ Cambridge Health Alliance, USA \\ ${ }^{3}$ Commonwealth Mental Health and Wellness Center, USA
}

Correspondence: Loretta Butehorn, Boston MA is a licensed psychologist and certified homeopath. She consults to Massachusetts Dept of Public Health, USA,

Email Ibutehornphd@gmail.com

Received: February 07, 2017 | Published: April 24, 2017

are highly diluted natural substances. As an example, homeopathic coffee (Coffea) is an effective remedy for someone who cannot sleep at night because their mind is too excited; what someone using a material dose of coffee might experience. Provings demonstrate when the homeopathic remedy Coffea is given to a person not having sleep symptoms sleeplessness occurs.

In the field of complementary and alternative medicine (CAM), homeopathy falls within the broad category of both alternative medicine systems and energy medicine.

\section{Patients and methods}

There were three cohorts of clients, all women ages 18-70, of varied ethnic backgrounds, all with long-term addiction to multiple recreational and prescription drugs. The first two cohorts were women living at a 6month residential substance treatment program in Boston. In each of these cohorts, clients self-selected to take or not take a single dose of homeopathic Nux vomica 200c after receiving information as to its benefit to reduce cravings. The third cohort was in another one month long residential treatment center in Boston with similar demographics and exact same procedure. Again clients selfselected to take or not a single dose of Nux vomica 200c.

\section{Nux vomica peparation}

Homeopathic Nux vomica 200c was prepared by Highland Homeopathic Labs under standard FDA guidelines.

Homeopathic medicines are prepared by taking a solution of the concentrated ingredient (mother tincture) and serially diluting it many times with succession/shaking at each step (potentization). A 200 dilution is known as 200 c potency; it is unlikely that even a single molecule of the mother tincture remains. 
The mother tincture is extracted from the seeds of Strychnos Nux vomica, a tree of the Loaniacae family. The general public uses Nux vomica as a treatment for over indulgence of alcohol exhibiting symptoms of headache, nausea, digestive upset, increased irritability and sensitivity to noise, light, and odors.

\section{Results}

In 2006-2008 we ran a homeopathic substance abuse clinic at women's substance abuse treatment center in Boston MA. This was a post-detoxification residential program for women ages 18-70 who were addicted to multiple drugs (alcohol, heroin, cocaine, meth etc.) It was a six month program for women to stabilize their recovery, develop life skills, reunite with their children and move out to a stable home, job and recovery.

The women arrived at the treatment program after 3-5days of medical detoxification. Most often these women were still suffering from Post Acute Withdrawal Syndrome (PAWS) with symptoms of cravings for drugs, irritability, mental confusion, low energy, sleep problems and lingering detox symptoms such as diarrhea, stomach upset etc. Cravings and general discomfort post detox typically result in early dropout from treatment and relapse into further drug use.

Using research from animal study, ${ }^{1}$ knowledge of homeopathic Materia Medical and the homeopathic historical use of Nux Vomica for symptoms of hangover relief and alcohol problems, we instituted a protocol using a genus epidemicus model of treatment, that is identification of a remedy based on the collective signs and symptoms of a group rather than personalized individuated case analysis. ${ }^{2}$ All clients entering the program were offered a single dose of Nux Vomica 200c after briefly being introduced to homeopathy and our understanding of its usefulness in reducing cravings for alcohol and other drugs.

Since drop out during early phases of treatment due to drug cravings is common, our goal was to reduce PAWS and stabilize the women enough that they could complete the treatment program (six months for first and second cohorts, one month for third cohort) and thus increase the likelihood of continued sobriety.

As this was a treatment program not a research project, data was kept but there was little in the way of analysis. Our rough data with two separate cohort groups (2006 and 2007) showed that women who chose the homeopathic protocol (it was a self-selected sample) completed the program at a percentage of $66 \%$ (for first cohort) and $63 \%$ (for second cohort) versus $33 \%$ and $37 \%$ for those who did not choose homeopathy . Both cohorts were women between 18 and 70, a wide range of ethnicity, and all multiple drug users with long histories. A very promising finding.

In Dec 2010, we were invited to open a similar homeopathic clinic at a large publicly funded treatment center at a Boston area substance abuse treatment program. This was a 28 day program-a much shorter time with extremely high dropout rates. We again instituted the same protocol. This time we were able to have their staff statistician compile the data (see chart.) Demographics for client population were again similar to previous groups.

With only 3months of data (mid Dec 2010-Mid Feb 2011) and a small sample ( $\mathrm{n}=18$ using homeopathy, 43 non-using), the results show a similar trend. There were some other interesting comparisons with those Not using homeopathy leaving against clinical advise shortly after entry at a higher percentage than those using homeopathy; and those discharged for non compliance (i.e. fighting, flagrant violation of program rules and the like) at a higher percentage than those using homeopathy.

A full year of clients and analysis (Dec 2010-Dec 2011) (see chart) continued to show the same trend of successful completion of treatment. A significant number of clients using homeopathy $(\mathrm{n}=138)$, $63 \%$, completed treatment as compared to those who did not select homeopathy ( $\mathrm{n}=130) 42 \%$.

\begin{tabular}{llllllll}
\hline Relapse & $\begin{array}{l}\text { ACA *Before 48 } \\
\text { hrs in Program }\end{array}$ & $\begin{array}{l}\text { Unknown } \\
\text { Disappearance }\end{array}$ & $\begin{array}{l}\text { Dismissed for } \\
\text { non Compliance }\end{array}$ & $\begin{array}{l}\text { Med/psych } \\
\text { Discharge }\end{array}$ & $\begin{array}{l}\text { ACA After 48 } \\
\text { hrs in Program }\end{array}$ & Still in Rx & $\begin{array}{l}\text { Completed } \\
\text { Successfully }\end{array}$ \\
\hline $0 \%$ & $11 \%$ & $7 \%$ & $6 \%$ & $3 \%$ & $23 \%$ & $7 \%$ & $42 \%$ \\
\hline
\end{tabular}

Non homeopathic group $\mathrm{n}=184$ Dec 20 I0-Dec 201 I Cohort 3-full year data *against clinical advise

\begin{tabular}{llllllll}
\hline Relapse & $\begin{array}{l}\text { ACA before } \\
\mathbf{4 8} \text { hrs }\end{array}$ & $\begin{array}{l}\text { Unknown } \\
\text { disappearance }\end{array}$ & $\begin{array}{l}\text { Dismissed for } \\
\text { non compliance }\end{array}$ & $\begin{array}{l}\text { Med/psych } \\
\text { bischarge }\end{array}$ & $\begin{array}{l}\text { ACA after } \\
\mathbf{4 8} \text { hrs }\end{array}$ & Still in Rx & $\begin{array}{l}\text { Completed } \\
\text { successfully }\end{array}$ \\
\hline $2 \%$ & $7 \%$ & $2 \%$ & $2 \%$ & $4 \%$ & $15 \%$ & $4 \%$ & $63 \%$ \\
\hline
\end{tabular}

Homeopathically treated group $n=\mid 38$ Dec 2010-Dec 201 I Cohort 3-full year data.

\section{Conclusion}

Despite the sample being self-selected and the data from the first two cohorts being informally collected as it was a clinic and not focused on research, the trend of a similar percentage of successful treatment completion of those using homeopathic treatment compared to those who did not warrants further study.

\section{Acknowledgments}

None.

\section{Conflicts of interest}

Author declares there are no conflicts of interest.

\section{Funding}

None.

\section{References}

1. Sukul NC, Ghosh S, Sinhasbabu SP, et al. Strychnos Nuv Vomica extract and its ultra high dilution reduce voluntary ethanol intake in rat. J Altern Com Med. 2001;7(2):187-193

2. van der Zee H Johannes C. "Healing Collective Trauma," in Homeopathy and Mental Health: Integrative Practice, Principles and Researc. Neatherlands: Homeolinks Publishers; 2010. 